Supplement of Geosci. Model Dev., 12, 4099-4113, 2019

https://doi.org/10.5194/gmd-12-4099-2019-supplement

(C) Author(s) 2019. This work is distributed under

the Creative Commons Attribution 4.0 License.

(c) (1)

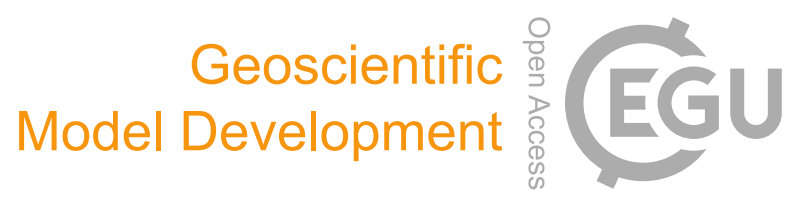

Supplement of

\title{
Evaluation of lossless and lossy algorithms for the compression of scientific datasets in netCDF-4 or HDF5 files
}

Xavier Delaunay et al.

Correspondence to: Xavier Delaunay (xavier.delaunay@thalesgroup.com)

The copyright of individual parts of the supplement might differ from the CC BY 4.0 License. 
This supplement details the commands and datasets necessary to reproduce the results tabulated in the paper.

\section{The Digit Rounding algorithm}

The table below provides tabulated values for the approximation of $\log _{10}\left(m_{i}\right)$ in the implementation of the Digit Rounding algorithm. Only 5 tabulated values are used in our implementation, enough to provide a good precision. The tabulated $v$ values for $\log _{10}\left(m_{i}\right)$ are such that $v \leq \log _{10}\left(m_{i}\right)$.

Table 1: Tabulated values for the approximation of $\log _{10}\left(m_{i}\right)$ in the implementation of the Digit Rounding algorithm

\begin{tabular}{cc}
\hline$m_{i}$ & $\begin{array}{c}\text { Approximated value } v \\
\text { for } \log _{10}\left(m_{i}\right)\end{array}$ \\
\hline $0.5 \leq m_{i}<0.6$ & -0.301029996 \\
$0.6 \leq m_{i}<0.7$ & -0.221848749 \\
$0.7 \leq m_{i}<0.8$ & -0.154901959 \\
$0.8 \leq m_{i}<0.9$ & -0.096910013 \\
$0.9 \leq m_{i}<1.0$ & -0.045757490 \\
\hline
\end{tabular}

15

The results reported in Table 2 and Table 3 of the paper can be reproduced compiling the Digit Rounding software in test and debug mode with the following command lines:

cd digiround/

make clean

20 make test $\mathrm{DEBUG}=1$

The results reported in Table 4 can be reproduced using the following script:

for nsd in \$(seq 7); do 
done

\section{Synthetic datasets}

Synthetic datasets with known statistics have been generated in order to test the compression algorithms under variable conditions. The following datasets have been generated:

- $\quad s 1$ a noisy sinusoid of 1 dimension,

- $\quad$ s3D a noisy sinusoid pulse of 3 dimensions.

10 The signal $s 1$ is a noisy sinusoid defined by:

$s 1(i)=c+a_{1} \times \sin \left(2 \pi i \frac{f_{s 1}}{f_{s}}\right)+n(i)$

Where $c$ is the mean value, $a_{1}$ is the amplitude of the sinusoid, $f_{s 1}$ is its frequency and $n(i)$ is a zero mean Gaussian noise of variance 1 . The signal $s 1$ is generated with $c=100, a_{1}$ computed so as to obtain a SNR of $20 \mathrm{~dB}$, and $\frac{f_{s 1}}{f_{s}}=\frac{17}{19 \times 2}$. It allows having a bit more than two samples per period with a pattern reproduced every 17 periods. It is generated over $N=2^{20}$ float sample values, each float value being encoded on 32 bits. The volume of the dataset $s 1$ is $4 \mathrm{MB}$. The dataset and its histogram

15 are shown in Fig. 1.

The signal $s 3 D$ a noisy sinusoid pulse of 3 dimensions defined by:

$s 3 D\left(i_{1}, i_{2}, i_{3}\right)=a_{2} \times \frac{\sqrt{i_{1}^{2}+i_{2}{ }^{2}+i_{3}{ }^{2}}}{\sqrt{L^{2}+M^{2}+N^{2}}} \times \sin \left(2 \pi \sqrt{i_{1}{ }^{2}+i_{2}{ }^{2}+i_{3}{ }^{2}} \frac{f_{s 3 D}}{f_{\text {ech }}}\right)+n\left(i_{1}, i_{2}, i_{3}\right)$

Where $L, M, N$ are the 3 dimensions of the signal $s 3 D, a_{2}$ is the amplitude of the sinusoid, $f_{s 3 D}$ is its frequency and $n\left(i_{1}, i_{2}, i_{3}\right)$ is a zero mean Gaussian noise of variance 1

The signal $s 3 D$ is generated with $L=256, M=256, N=2048, a_{2}$ computed to obtain a SNR of $40 \mathrm{~dB}$, and $\frac{f_{S 3 D}}{f_{S}}=\frac{17 \times 8}{19 \times N}$ in

20 order to have 4 periods on the main axis. It is generated over $L \times M \times N=2^{27}$ float sample values, each float value being encoded on 32 bits. The volume of the dataset $s 3 D$ is $512 \mathrm{MB}$. The dataset and its histogram are shown in Fig. 2.

The datasets $s 1$ and $s 3 D$ have been stored into netCDF-4 formatted files. 

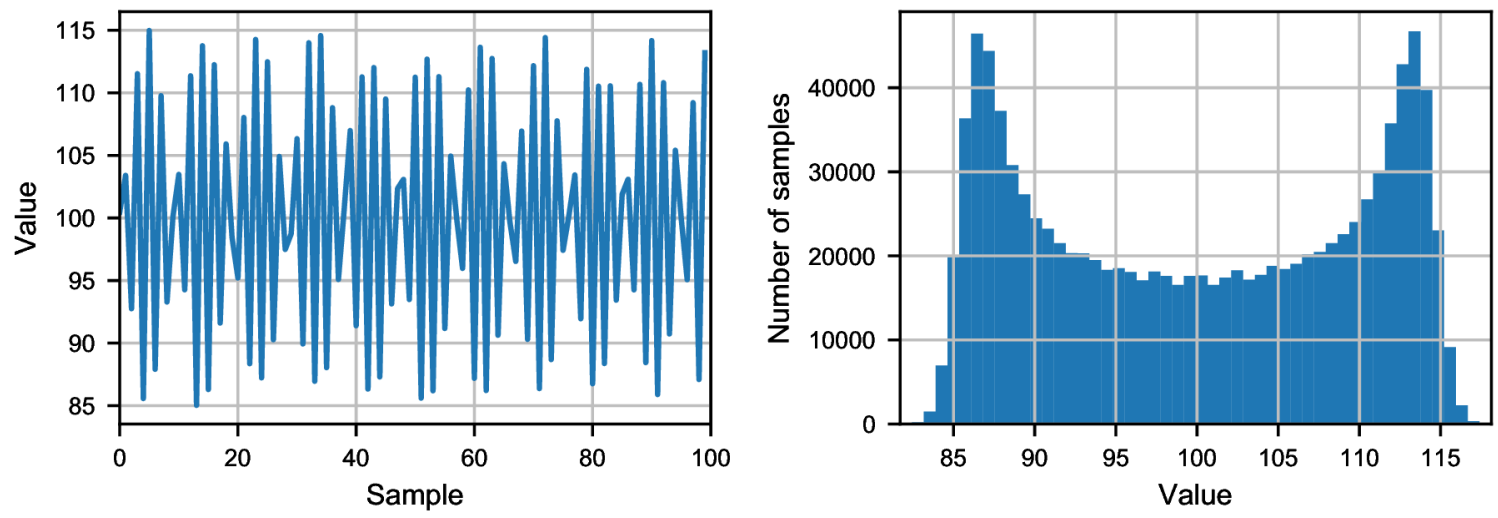

Figure 1: First 100 samples of the dataset $s 1$ (left) and histogram of the sample values (right).
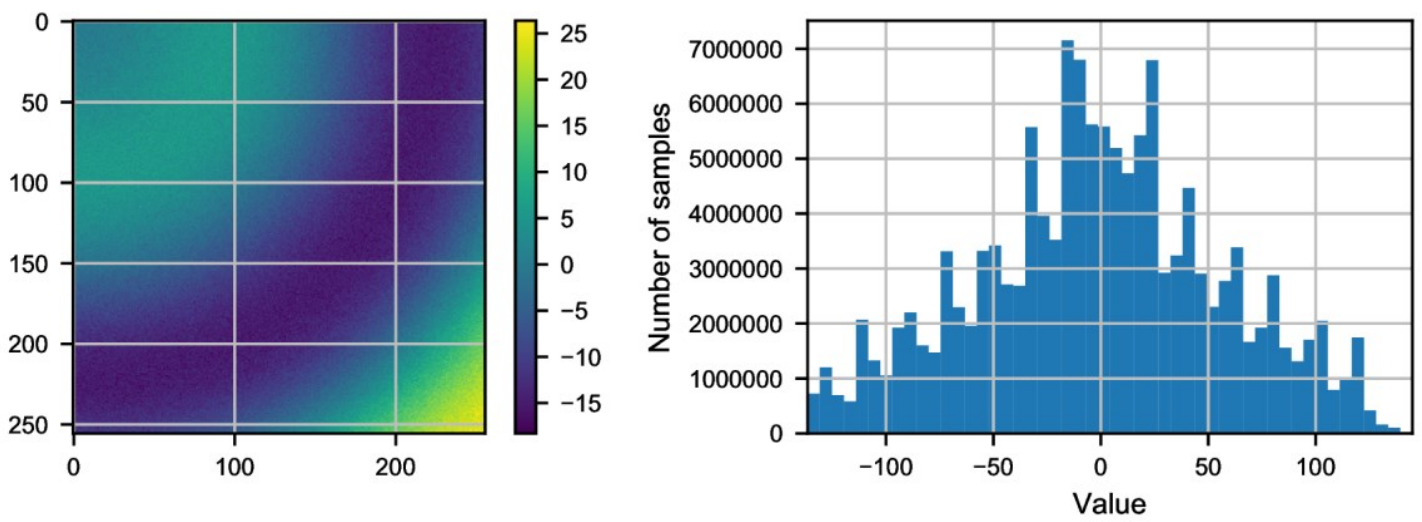

Figure 2: Representation of the first slices $s 3 D\left(i_{1}, i_{2}, 0\right)$ (left), and histogram of the sample values (right).

\section{Performance assessment of lossless compression methods}

We ran lossless compression algorithm using h5repack tool from the HDF5 library in version 1.8.19, Deflate implemented in zlib 1.2.11, Zstandard in version 1.3.1 with the corresponding HDF5 filter available on the HDF web portal (http://portal.hdfgroup.org/display/support/Filters), and the implementation of LZ4 and Bitshuffle in the python package Bitshuffle-0.3.4. The compression is performed calling h5repack tool with a command line formatted as follows:

h5repack -i in_file.nc -o compressed_file.h5 [--filter=var:params]

where in file.nc is the input dataset formatted as a netCDF-4 file and compressed_file.h5 is the compressed dataset in HDF5 file format. The input dataset contains the var variable processed by one or several HDF5 filter. Each HDF5 filter is identified by a unique ID: 32015 for Zstandard and 32008 for Bitshuffle. The following table provides the list of filter options used. They shall replace the filter option between brackets on previous command line. 
Table 2: Command lines and parameters used for the compression with h5repack.

\begin{tabular}{lll}
\hline Compression algorithms & Command line & Parameters \\
\hline Deflate & --filter=var:GZIP=dfl_lvl & dfl_lvl from 1 to 9 \\
Shuffle + Deflate & --filter=var:SHUF --filter=var:GZIP=dfl_lvl & dfl_lvl from 1 to 9 \\
Zstandard & --filter=var:UD=32015,1,zstd_lvl & zstd_lvl from 1 to 22 \\
Shuffle + Zstandard & --filter=var:SHUF --filter=var:UD=32015,1,zstd_lvl & zstd_lvl from 1 to 22 \\
Bitshuffle + Zstandard & --filter=var:UD=32008,1,1048576 --filter=var:UD=32015,1,zstd_lvl & zstd_lvl from 1 to 22 \\
Bitshuffle + LZ4 & --filter=var:UD=32008,2,1048576,2 & - \\
\hline
\end{tabular}

The decompression has been performed calling h5repack tool with a command line formatted as follows:

h5repack -i compressed_file.h5 -o out_file.h5 --filter=var:NONE

5 Compression and decompression has been performed on a Dell T1600 with an Intel Xeon E31225 4 cores CPU at 3.1GHz, and 4GB memory under RedHat 6.5 (64 bits) OS. Compression and decompression were run on a single core.

\section{Performance assessment of lossy compression methods}

\section{Performance comparison in the absolute error bounded compression mode}

The command lines provided in this section allow reproducing the results provided in Table 5 of the paper.

10 Sz compression is performed calling h5repack tool with the following command lines:

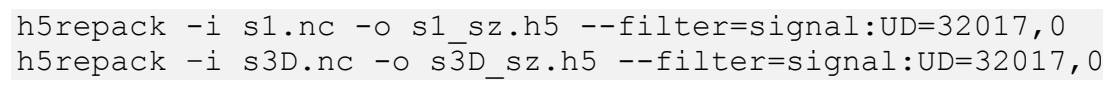

Sz compression filter is identified by its ID (32017) provided on the command line. The following "0" is the number of filter parameters. In the case of $\mathrm{Sz}$, the filter does not have any parameter to set. That is why there are 0 parameters. Sz is 15 configured via the sz.config file located in the directory from where h5repack is called. sz.config file content is reproduced below:

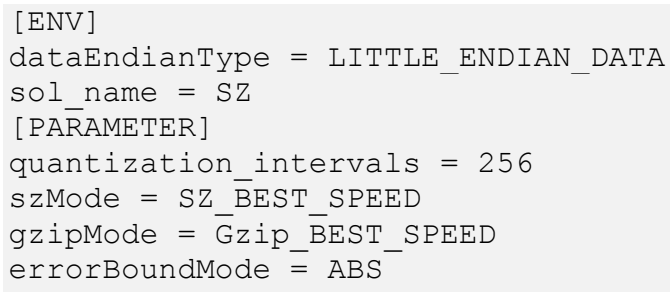

Decimal Rounding is performed calling the $n c k s$ tool from NCO toolkit. The algorithm is run with the following command lines (note the period before the $d s d$ parameter): 
ncks $-0-4-\mathrm{L} 1--\mathrm{ppc}$ signal=.0 s1.nc s1_bg.nc

ncks $-0-4-\mathrm{L} 1--\mathrm{ppc}$ signal=.0 s3D.nc s $\overline{3} \mathrm{D} \_\mathrm{bg} . \mathrm{nc}$

\section{Performance comparison in the relative error bounded compression mode}

\section{Dataset s1}

5 The command lines provided in this section allow reproducing the results provided in Table 6 of the paper.

Sz compression is performed calling h5repack tool with a command line formatted as follows:

h5repack -i s1.nc -o s1_sz.h5 --filter=signal:UD=32017,0

Sz compression is configured via the sz.config file located in the directory from where h5repack is called. sz.config file content is reproduced below:

$10 \quad[\mathrm{ENV}]$

dataEndianType = LITTLE ENDIAN DATA

sol_name $=$ SZ

[PARAMETER]

quantization intervals $=256$

15 szMode $=$ SZ DEFAULT COMPRESSION

gzipMode $=\bar{G}$ zip_BEST_SPEED

errorBoundMode $=\mathrm{PW} \overline{\mathrm{R}} \mathrm{LL}$

pw_relBoundRatio $=\overline{0} .00424$

pwr_type $=$ MAX

20 Bit Grooming is performed calling the $n c k s$ with the following command lines:

ncks $-0-4-\mathrm{L} 1--\mathrm{ppc}$ signal=3 s1.nc s1_bg_3.nc

ncks -0 -4 -L 1 --ppc signal=2 s1.nc s1_bg_2.nc

Digit Rounding is performed calling h5repack tool with the following command line:

h5repack -i s1.nc -o s1 dr 3.h5 --filter=signal:UD=47987, 1, 1, 3

--filter=signal: $\overline{S H U F}--f i l$ ter=signal $:$ GZIP $=1$

\section{Dataset s3D}

The command lines provided in this section allow reproducing the results provided in Table 7 of the paper.

Sz compression is performed calling h5repack tool with a command line formatted as follows:

h5repack -i s3D.nc -o s3D_sz.h5 --filter=signal:UD=32017,0

$30 \mathrm{Sz}$ compression is configured via the sz.config file located in the directory from where h5repack is called. sz.config file content is reproduced below:

[ENV]

dataEndianType $=$ LITTLE_ENDIAN_DATA

sol name $=\mathrm{SZ}$

35 [PARAMETER]

quantization intervals $=256$

szMode = SZ DEFAULT COMPRESSION

gzipMode $=\overline{\mathrm{G} z i p}$ _BEST_SPEED 
Bit Grooming is performed calling the $n c k s$ with the following command lines:

5 ncks $-0-4 \quad-$ L $1--p p c$ signal=3 s3D.nc s3D_bg $3 . n c$

ncks - 0 -4 - - 1 1 --ppc signal=2 s3D.nc s3D_bg_2.nc

Digit Rounding is performed calling h5repack tool with the following command line:

h5repack -i s3D.nc -o s3D_dr_3.h5 --filter=signal:UD=47987, 1, 1, 3 ।

--filter=signal:SHUF --filter=signal:GZIP=1

10

We used two version of the same dataset:

- TMP_TEST_SWI_L1A F_20160830T150000_20160830T164500.nc is the 'raw' dataset.

- CFO_TEST_SWI_L1A F_20160830T150000_20160830T164500.nc is the same dataset after clipping and Shuffle + Deflate (3) compression.

15 Table 3 provides the least significant digit $(l s d)$ of each variable of this dataset.

Table 3: Variables and least significant digit $(l s d)$ in CFOSAT dataset.

\begin{tabular}{ll|ll|ll|ll}
\hline Variable & $l s d$ & Variable & $l s d$ & Variable & $l s d$ & Variable & $l s d$ \\
\hline altitude & 3 & elevation_0 & 5 & lat_11a_0 & 3 & phi_geo & 3 \\
cal_ratio_0 & 3 & elevation_1 & 5 & lat_11a_1 & 3 & pri & 1 \\
cal_ratio_1 & 3 & elevation_2 & 5 & lat_11a_2 & 3 & projected_velocity 3 \\
cal_ratio_2 & 3 & elevation_3 & 5 & lat_11a_3 & 3 & pseudo_misp & 14 \\
cal_ratio_3 & 3 & elevation_4 & 5 & lat_11a_4 & 3 & radar_range_0 & 3 \\
cal_ratio_4 & 3 & elevation_5 & 5 & lat_11a_5 & 3 & radar_range_1 & 3 \\
cal_ratio_5 & 3 & ground_range_0 & 3 & lon_11a_0 & 3 & radar_range_2 & 3 \\
cycle_duration & 7 & ground_range_1 & 3 & lon_11a_1 & 3 & radar_range_3 & 3 \\
earth_radius & 3 & ground_range_2 & 3 & lon_11a_2 & 3 & radar_range_4 & 3 \\
echo_11_0 & 12 & ground_range_3 & 3 & lon_11a_3 & 3 & radar_range_5 & 3 \\
echo_11_0_nt & 12 & ground_range_4 & 3 & lon_11a_4 & 3 & & \\
echo_11_0_stdnt & 12 & ground_range_5 & 3 & lon_11a_5 & 3 & \\
echo_11a_0 & 3 & incidence_0 & 5 & ly & 2 & \\
echo_11a_1 & 3 & incidence_1 & 5 & mispointing & 14 & \\
echo_11a_2 & 3 & incidence_2 & 5 & nesig0 & 3 & \\
echo_11a_3 & 3 & incidence_3 & 5 & orbital_velocity & 3 & \\
echo_11a_4 & 3 & incidence_4 & 5 & phi & 3 & \\
echo_11a_5 & 3 & incidence_5 & 5 & phi_azimuth & 3 & & \\
\hline
\end{tabular}


The following command lines allow reproducing the results provided in Table 8 of the paper.

We first extract the ground_range_5 variable from the 'clipped' dataset and decompress it:

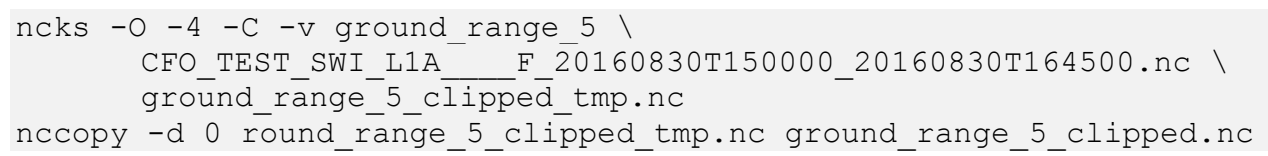

Then, CFOSAT Clipping + Shuffle + Deflate (3) compression is performed using the following command line:

nccopy -s -d 3 ground_range_5_clipped.nc ground_range_5_clipped_dfl3.nc

CFOSAT Clipping + Zstd (2) compression is performed using the following command line:

10 nccopy -F "ground_range_5,32015,2" ground_range_5_clipped.nc ground_range_5_clipped_zstd2.nc

For the other compression methods, we first extract the ground_range_5 variable from the 'raw' dataset:

$\begin{aligned} \text { ncks } & -0-4-\mathrm{C}-\mathrm{V} \text { ground_range 5 I } \\ & \text { TMP_TEST_SWI_L1A_E_E_20160830T150000_20160830T164500.nc ground_range_5.nc }\end{aligned}$

Then, Sz (absErrBound = 1e-3, Gzip_BEST_SPEED) compression is performed using the following command line after

15 having correctly configured the sz.config file:

h5repack --filter=ground_range_5:UD=32017,0 -i ground_range_5.nc -o ground_range_5_sz.h5

Decimal Rounding $(d s d=.3)+$ Shuffle + Deflate (1) compression is performed using the following command line:

ncks -0 -4 -L 1 --ppc ground_range_5=.3 ground_range_5.nc ground_range_5_dr.nc

Bit Grooming ( $n s d=8)+$ Shuffle + Deflate (1) compression is performed using the following command line:

20 ncks -0 -4 - - 1 --ppc ground_range_5=8 ground_range_5.nc ground_range_5_bg.nc

Digit Rounding $(n s d=8)+$ Shuffle + Deflate (1) compression is performed using the following command line:

h5repack h5 --filter=ground_range_5:UD=47987,1,1,8--filter=ground_range_5:SHUF ।

--filter=ground_range_5:GZIP $=1$-i ground_range_5.nc-o ground_range_5_dr.h5

25 The following command lines allow reproducing the results provided in Table 9 of the paper.

We first decompress the 'clipped' dataset:

nccopy -d 0 CFO_TEST_SWI_L1A_F_20160830T150000_20160830T164500.nc I CFO_TEST_SWI_L1A_E_E_20160830T150000_20160830T164500_decompressed.nc

Then, CFOSAT Clipping + Shuffle + Deflate (3) compression is performed using the following command line:

nccopy -s -d 3 CFO_TEST_SWI_L1A_F_20160830T150000_20160830T164500_decompressed.nc। CFO_TEST_SWI_L1A F_20160830T150000_20160830TT164500_df13.nc

CFOSAT Clipping + Shuffle + Zstd (2) compression is performed using the following command line: 


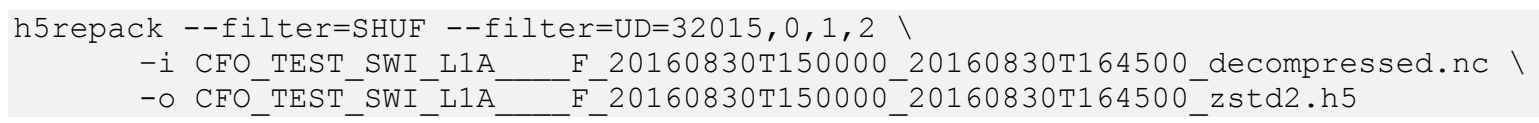

For the other compression methods, we used the 'raw' dataset.

5 Decimal Rounding + Shuffle + Deflate (1) compression is performed using the following command line, setting the $d s d$ parameter on a per variable basis and corresponding to the $l s d$ parameters of CFOSAT clipping (see Table 3):

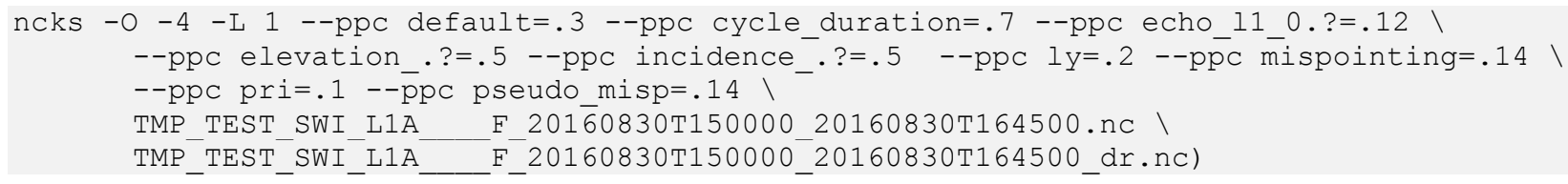

Bit Grooming (nsd = 8) + Shuffle + Deflate (1) compression is performed using the following command line:

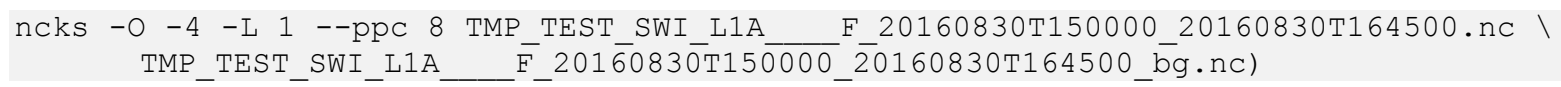

15 Digit Rounding $(n s d=8)+$ Shuffle + Deflate (1) compression is performed using the following command line:

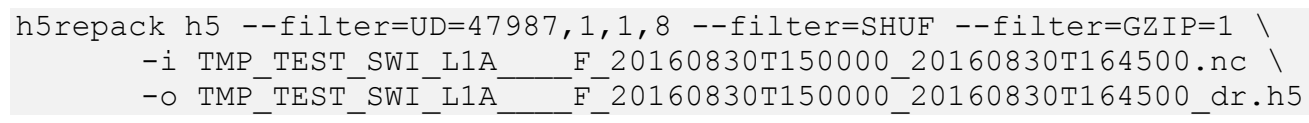

Sz compression has been applied variable per variable using the following command lines:

20 \# Get the list of variables in the dataset

var_list $=\$($ h5ls TMP_TEST_SWI_L1A___E_20160830T150000_20160830T164500.nc I awk '\{print \$1\}')

\# Loop over the varíables

for var in \$var_list;

do

\section{SWOT datasets}

We used two different SWOT datasets:

- SWOT_L2_HR_PIXC_000_210_46N-R_main.nc is a simplified simulated SWOT L2_HR_PIXC pixel cloud product;

- $\quad$ pixel_cloud.nc is a realistic and representative SWOT L2 pixel cloud product 
Table 4 provides the precision in number of significant digits ( $n s d)$ required for each variable of the SWOT_L2_HR_PIXC_000_210_46N-R_main.nc dataset.

Table 4: Precision in number of significant digits (nsd) required for each variable of the SWOT_L2_HR_PIXC_000_210_46NR_main.nc dataset.

\begin{tabular}{ll|ll|ll}
\hline Variable & $n s d$ & Variable & $n s d$ & Variable & $n s d$ \\
\hline azimuth_index & 4 & dlook_dphase_z & 6 & look_unit_x & 8 \\
classification & 3 & dphase & 6 & look_unit_y & 8 \\
coherent_power & 8 & dry_tropo_range_correction & 4 & look_unit_z & 8 \\
continuous_classification & 8 & height & 6 & num_rare_looks & 2 \\
cross_track & 15 & ice_flag & 8 & phase_screen & 6 \\
dark_water_flag & 8 & ifgram_imag & 15 & pixel_area & 11 \\
delta_x & 8 & ifgram_real & 15 & power_left & 8 \\
delta_y & 8 & illumination_time & 15 & power_right & 8 \\
delta_z & 8 & instrument_attitude_correction & 6 & range_index & 4 \\
dheight_dbaseline & 6 & instrument_baseline_correction & 6 & reference_layover_flag & 8 \\
dheight_dphase & 8 & instrument_phase_correction & 6 & reference_layover_height_error & 8 \\
dheight_drange & 6 & instrument_range_correction & 6 & sensor_s & 11 \\
dheight_droll & 8 & ionosphere_range_correction & 6 & solid_earth_tide_height_correction & 4 \\
dlook_dphase_x & 6 & latitude & 15 & wet_tropo_range_correction & 4 \\
dlook_dphase_y & 6 & longitude & 15 & xover_roll_correction & 4 \\
\hline
\end{tabular}

Table 5 provides the precision in number of significant digits $(n s d)$ required for each variable of the pixel_cloud.nc dataset.

Table 5: Precision in number of significant digits (nsd) required for each variable of the pixel_cloud.nc dataset.

\begin{tabular}{ll|ll|ll}
\hline Variable & $n s d$ & Variable & $n s d$ & Variable & $n s d$ \\
\hline azimuth_index & 4 & ifgram & 7 & power_left & 8 \\
classification & 3 & illumination_time & 15 & power_right & 8 \\
coherent_power & 8 & incidence_angle & 8 & range_index & 4 \\
continuous_classification & 8 & latitude & 15 & regions & 7 \\
cross_track & 15 & longitude & 15 & sigma0 & 7 \\
dheight_dphase & 8 & num_med_looks & 3 & x_factor_left & 7 \\
dlatitude_dphase & 5 & num_rare_looks & 2 & x_factor_right & 7 \\
dlongitude_dphase & 5 & phase_noise_std & 7 & & \\
height & 6 & pixel_area & 11 & & \\
\hline
\end{tabular}


The following command lines allow reproducing the results provided in Table 10 of the paper.

We first extract the height variable from SWOT dataset and decompress it:

ncks $-0-v$ height SWOT_L2_HR_PIXC_000_210_46N-R_main.nc height_tmp.nc

nccopy -d 0 height_tmp.nc height. ñc

5 Then, Shuffle + Deflate (4) compression is performed using the following command line:

nccopy -s -d 4 height.nc height_dfl4.nc

Shuffle + Zstd (2) compression is performed using the following command line:

h5repack --filter=height:SHUF --filter=height:UD=32015,1,1,2 ।

-i height.nc -o height_zstd2.h5

$10 \mathrm{Sz}\left(p w \_\right.$relBoundRatio $=5 e-6$, Gzip_BEST_SPEED) compression is performed using the following command line after having correctly configured the sz.config file:

h5repack --filter=ground_range_5:UD=32017,0 -i height.nc -o height_sz.h5

Bit Grooming ( $n s d=6)+$ Shuffle + Deflate (1) compression is performed using the following command line:

ncks -0 $-4-\mathrm{L} 1--\mathrm{ppc}$ height=6 height.nc height_bg.nc

15 Digit Rounding $(n s d=6)+$ Shuffle + Deflate (1) compression is performed using the following command line:

h5repack h5 --filter=height:UD=47987,1,1,6--filter=height:SHUF --filter=height:GZIP=1 । -i height.nc -o height_dr.h5

The following command lines allow reproducing the results provided in Table 11 of the paper.

20 We first extract the pixel_area variable from SWOT dataset and decompress it:

ncks -O -v pixel_area pixel_cloud.nc pixel_area_tmp.nc

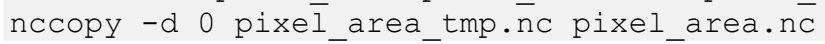

Then, Shuffle + Deflate (4) compression is performed using the following command line:

nccopy -s -d 4 pixel_area.nc pixel_area_dfl4.nc

25 Shuffle + Zstd (2) compression is performed using the following command line:

h5repack --filter=pixel_area:SHUF --filter=pixel_area:UD=32015, 1, 1,2
-i pixel_area.nc -o pixel_area_zstd2.h5

$\mathrm{Sz}(p w$ relBoundRatio $=5 e-9$, Gzip_BEST_SPEED) compression is performed using the following command line after having correctly configured the sz.config file:

30 h5repack --filter=ground_range_5:UD=32017,0 -i pixel_area.nc -o pixel_area_sz.h5

Bit Grooming $(n s d=11)+$ Shuffle + Deflate (1) compression is performed using the following command line:

ncks -0 -4 -L 1 --ppc pixel_area=11 pixel_area.nc pixel_area_bg.nc

Digit Rounding $(n s d=11)+$ Shuffle + Deflate (1) compression is performed using the following command line: 
The following command lines allow reproducing the results provided in Table 12 of the paper.

5 We first decompress SWOT dataset:

ncCopy -d 0 SWOT_L2_HR_PIXC_000_210_46N-R_main.nc SWOT_L2_HR_PIXC_decomp.nc

Then, Shuffle + Deflate (4) compression is performed using the following command line:

nccopy -s -d 4 SWOT_L2_HR_PIXC_decomp.nc SWOT_L2_HR_PIXC_dfI4.nC

Clipping + Shuffle + Zstd (2) compression is performed using the following command line:

h5repack --filter=SHUF --filter=UD=32015,0,1,2 ।

$$
\text { -i SWOT_L2_HR_PIXC_decomp.nc -o SWOT_L2_HR_PIXC_zstd2.h5 }
$$

Bit Grooming + Shuffle + Deflate (1) compression is performed using the following command line, setting the $n s d$ parameter on a per variable basis corresponding to the required precision (see Table 4):

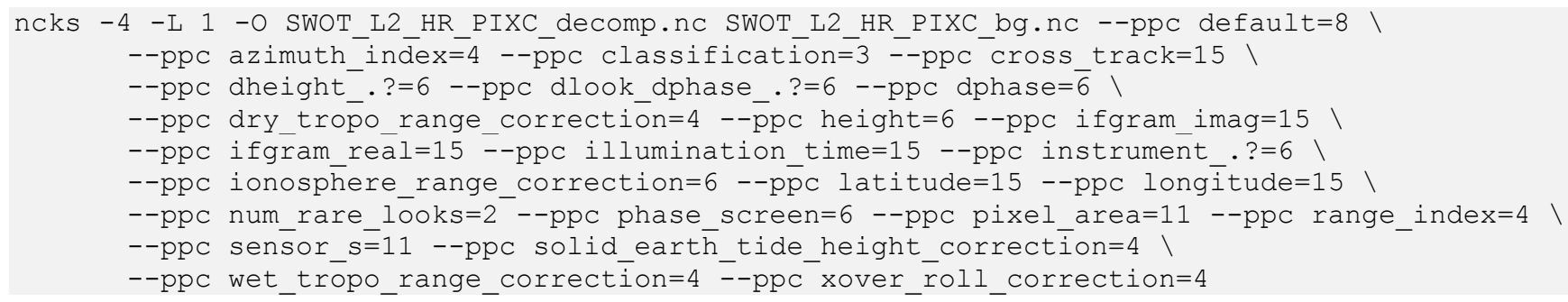

Digit Rounding + Shuffle + Deflate (1) compression is performed using the following command line, setting the $n s d$ parameter on a per variable basis corresponding to the required precision (see Table 4): filter=coherent_power:GZIP=1 --filter=continuous_classification:UD=47987,0,1,8 -filter=continuous classification:SHUF --filter=cōntinuous classification:GZIP=1 -filter=cross_track $: U D=47987,0,1,15$--filter=cross_track:SḦUF -filter=cross_track:GZIP=1 --filter=dark_water_flağ:UD=47987,0,1,8 -filter=dark water flag:SHUF --filter=dā̌k watēer flag:GZIP=1 - -

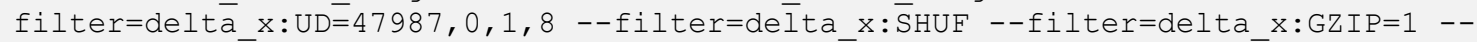
filter=delta_y:UD=47987,0,1,8 --filter=delta_y:SHUF --filter=delta_y:GZIP=1 -filter=delta z:UD=47987,0,1,8 --filter=delta z:SHUF --filter=delta z:GZIP=1 -filter=dheight_dbaseline:UD=47987,0,1,6 --filter=dheight_dbaseline:SHUF -filter=dheight_dbaseline:GZIP=1 --filter=dheight_dphase: ŪD=47987,0,1,8 -filter=dheight dphase:SHUF --filter=dheight dphase:GZIP=1 - filter=dheight drange: $U D=47987,0,1,6$--filtēer=dheight drange:SHUF -filter=dheight_drange:GZIP=1 --filter=dheight_droll:UD $=47987,0,1,8$-filter=dheight_droll:SHUF --filter=dheight_droll:GZIP=1 -filter=dlook_dphase_x:UD=47987,0,1,6 --filter=dlook dphase $x:$ SHUF -filter=dlook_dphase_x:GZIP=1 --filter=dlook_dphase_y: UD=479̄87,0,1,6 -- 
filter=dlook_dphase_y:SHUF --filter=dlook_dphase_y:GZIP=1 -filter=dlook_dphase_z:UD=47987,0,1,6 --filter=dlook_dphase_z:SHUF --

filter=dlook_dphase_z:GZIP=1 --filter=dphase:UD=47987,0, $1, \overline{6}$--filter=dphase:SHUF -filter=dphase $:$ GZIP $=\overline{1}$--filter=dry_tropo_range_correction:UD=47987,0,1,4 --

filter=dry tropo range correction: SHUF --filtēedry tropo range correction:GZIP=1 -filter=height: UD=47987,0,1,6 --filter=height:SHUF --filter =height:GZIP=1 --

filter=ice_flag:UD=47987,0,1,8 --filter=ice_flag:SHUF --filter=ice_flag:GZIP=1 -filter=ifgram imag:UD=47987,0,1,15 --filter=ifgram imag: SHUF --

filter=ifgram_imag:GZIP=1 --filter=ifgram_real:UD=47987,0,1,15 --

filter=ifgram_real:SHUF --filter=ifgram_rēal:GZIP=1 --

filter=illumination time:UD $=47987,0,1,1 \overline{5}$-filter=illumination time: SHUF -

filter=illumination_time:GZIP=1 --filter=instrument_attitude_correction:UD=47987,0, 1, 6 --filter=instrument_attitude_correction:SHUF --

filter=instrument_attitude_correction:GZIP=1 --

filter=instrument_baseline_correction:UD $=47987,0,1,6$--

filter=instrument_baseline_correction:SHUF --

filter=instrument_baseline_correction:GZIP=1 - -

filter=instrument_phase_correction: UD=47987, 0, 1,6 --

filter=instrument_phase_correction:SHUF --filter=instrument_phase_correction:GZIP=1 -filter=instrument_range_correction:UD=47987,0,1,6 --

filter=instrument_range_correction:SHUF --filter=instrument_range_correction:GZIP=1 -filter=ionosphere_range_correction:UD=47987,0,1,6 --

filter=ionosphere_range_correction:SHUF --filter=ionosphere_range_correction:GZIP=1 -filter=latitude: $\mathrm{U} \overline{\mathrm{D}}=4798 \overline{7}, 0,1,15$--filter=latitude:SHUF --fī̄ter=lātitude:GZIP=1 --

filter=longitude:UD=47987,0,1,15 --filter=longitude:SHUF --filter=longitude:GZIP=1 --

filter=look unit $\mathrm{x}: \mathrm{UD}=47987,0,1,8$--filter=look unit $\mathrm{x}:$ SHUF --

filter=look_unit_x:GZIP=1 --filter=look_unit_y: $\bar{U} D=47 \overline{9} 87,0,1,8$--

filter=look_unit_y:SHUF --filter=look_uñit_y:GZIP=1 --

filter=look_unit_z:UD=47987,0,1,8 --filter=look_unit_z:SHUF --

filter=look_unit_z:GZIP=1 --filter=num_rare_looks:UD=47987,0,1,2 - -

filter=num_rare_looks:SHUF --filter=num_rare_looks:GZIP=1 --

filter=phase_screen:UD=47987,0,1,6 --filtter=phase_screen:SHUF --

filter=phase_screen:GZIP=1 --filter=pixel_area:UD=47987,0,1,11 --

filter=pixel_area:SHUF --filter=pixel_areáa:GZIP=1 --filter=power_left:UD=47987,0,1,8 -filter=power_left:SHUF --filter=power_left:GZIP=1 --filter=power_right:UD=47987,0,1,8 --filter=power__right:SHUF --filter=power_right:GZIP=1 --

filter=range_iñdex:UD=47987,0,1,4 --filtēer=range_index:SHUF --

filter=range_index:GZIP=1 --filter=reference_layover_flag:UD=47987,0, 1,8 --

filter=reference_layover_flag:SHUF --filter=referencé_layover_flag:GZIP=1 - -

filter=reference_layover_height_error:UD $=47987,0,1,8$--

filter=reference_layover_height_error:SHUF --

filter=reference_layover_height_error:GZIP=1 --filter=sensor_s:UD=47987,0,1,11 -filter=sensor s: SHUF --fílter=sensor s:GZIP=1 --

filter=solid_earth_tide_height_correction:UD $=47987,0,1,4 \ldots$

filter=solid_earth_tide_height_correction:SHUF --

filter=solid_earth_tide_height_correction:GZIP=1 --

filter=wet_tropo_range_correction: UD=47987,0,1,4 --

filter=wet_tropo_range_correction:SHUF --filter=wet_tropo_range_correction:GZIP=1 -filter=xover_roli__correction: UD=47987,0,1,4 --filter =xover_roll_correction:SHUF -filter=xover_roll_correction:GZIP=1)

The following command lines allow reproducing the results provided in Table 13 of the paper.

Shuffle + Deflate (4) compression is performed using the following command line:

nccopy -s -d 4 pixel_cloud.nc PIXEL_CLOUD_dfl4.nc 
Clipping + Shuffle + Zstd (2) compression is performed using the following command line:

h5repack --filter $=$ SHUF --filter=UD=32015,0,1,2 ।

-i pixel_cloud_decomp.nc -o pixel_cloud_zstd2.h5

Bit Grooming + Shuffle + Deflate (1) compression is performed using the following command line, setting the $n s d$ parameter

5 on a per variable basis corresponding to the required precision (see Table 5):

\begin{tabular}{|c|c|}
\hline & 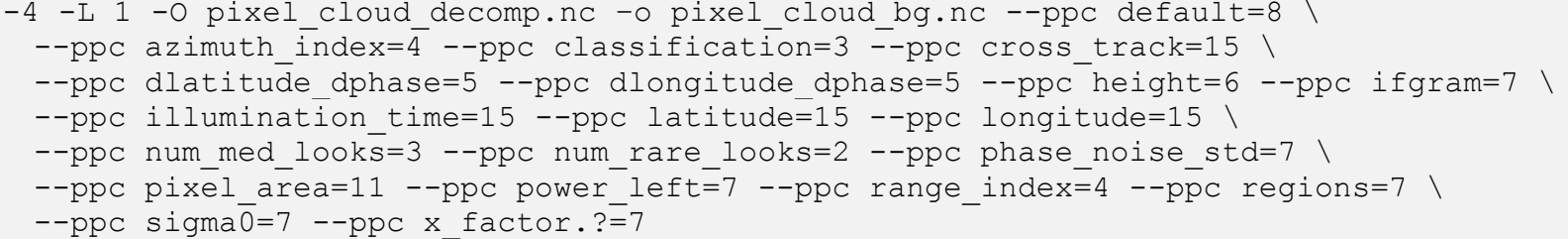 \\
\hline
\end{tabular}

Digit Rounding + Shuffle + Deflate (1) compression is performed using the following command line, setting the nsd parameter on a per variable basis corresponding to the required precision (see Table 5):

15

h5repack -i pixel_cloud_decomp.nc -o pixel_cloud_dr.h5 ।

--filter=azimuth_index:UD=47987,0,1,4 --filter=azimuth_index:SHUF --

filter=azimuth iñdex:GZIP=1 --filter=classification:UD=47987,0,1,3 _filter=classification:SHUF --filter=classification:GZIP=1 -filter=coherent_power: $U D=47987,0,1,8$--filter=coherent_power:SHUF -filter=coherent_power:GZIP=1 --filter=continuous_classification:UD=47987,0,1,8 -filter=continuous_classification:SHUF --filter=continuous_classification:GZIP=1 -filter $=$ cross track $: U D=47987,0,1,15$--filter=cross track: $\bar{H} U F$-filter=cross track:GZIP=1 --filter=dheight dphase: UD=47987,0,1,8 - filter=dheight_dphase:SHUF --filter=dheight_dphase:GZIP=1 - filter=dlatitude_dphase:UD=47987,0,1,5 --filter=dlatitude_dphase:SHUF -filter=dlatitude dphase:GZIP=1 --filter=dlongitude dphase:UD=47987,0,1,5 -filter=dlongitude_dphase:SHUF --filter=dlongitude_d̄phase:GZIP=1 -filter=height: $\mathrm{UD}=\overline{4} 7987,0,1,6$--filter=height:SHUF ${ }^{-}$--filter=height:GZIP=1 -filter=ifgram:UD=47987,0,1,7 --filter=ifgram:SHUF --filter=ifgram:GZIP=1 -filter=illumination_time: UD=47987,0,1,15--filter=illumination_time:SHUF -filter=illumination_time:GZIP=1 --filter=incidence_angle:UD=479̄87,0,1,8 -filter=incidence anğle:SHUF --filter=incidence angle:GZIP=1 -filter=latitude: $\bar{U} \mathrm{D}=47987,0,1,15$--filter=latitüde:SHUF --filter=latitude:GZIP=1 -filter=longitude:UD=47987,0,1,15 --filter=longitude:SHUF --filter=longitude:GZIP=1 -filter=num_med_looks:UD=47987,0,1,3--filter=num_med_looks:SHUF -filter=num_med_looks:GZIP=1 --filter=num_rare_loōks: $\bar{U} D=47987,0,1,2$-filter=num_rare_looks:SHUF --filter=num_rare_looks:GZIP=1 -filter=phašse_noīse_std:UD=47987,0,1,7 --filtēer=phase_noise_std:SHUF -filter=phase_noise_std:GZIP=1 --filter=pixel_area:UD=47987,0, 1,11 --

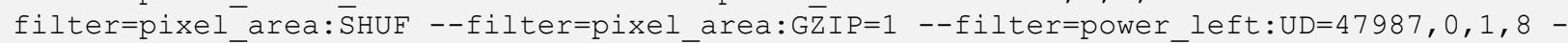
-filter=power_left:SHUF - -filter=power_left:GZIP=1 --filter=power_right:UD=47987,0,1,8 --filter=power_right:SHUF --filter=power_right:GZIP=1 --

filter=range iñdex:UD=47987,0,1,4 --filtêr=range index:SHUF -filter=range_index:GZIP=1 --filter=regions:UD=479̄87, 0, 1, 7 --filter=regions:SHUF -filter=regions:GZIP=1 --filter=sigma0:UD=47987,0,1,7 --filter=sigma0:SHUF -filter=sigma0:GZIP=1 --filter=x_factor_left:UD=47987,0,1,7 --filter=x_factor_left:SHUF --filter=x_factor_left:GZIP=1 --filter=x_factor_right:UD=47987,0,1,7 -filter=x_factor_right:SHUF --filter=x_factor_right:GZIP=1 


\section{Example usage with netCDF-4 tools}

From version 4.6.0 - January 24, 2018, netCDF supports HDF5 dynamic filters. It is now possible to make use of compression filters through nccopy tool. However, currently it supports only one filter: it does not yet allow chaining several filters such as Shuffle, Digit Rounding and Deflate. Nevertheless, below is provided an example of command line calling the 5 Digit Rounding algorithm with $n s d=3$ on the dataset ground_range_5_clipped.nc.

nccopy -F "ground_range_5,47987,3" ground_range_5_clipped.nc ground_range_5_clipped_dr.nc 